\title{
Control Lyapunov Functions and Hybrid Zero Dynamics
}

\author{
Aaron D. Ames, Kevin Galloway, and J. W. Grizzle
}

\begin{abstract}
Hybrid zero dynamics extends the Byrnes-Isidori notion of zero dynamics to a class of hybrid models called systems with impulse effects. Specifically, given a smooth submanifold that is contained in the zero set of an output function and is invariant under both the continuous flow of the system with impulse effects as well as its reset map, the restriction dynamics is called the hybrid zero dynamics. Prior results on the stabilization of periodic orbits of the hybrid zero dynamics have relied on input-output linearization of the transverse variables. The principal result of this paper shows how control Lyapunov functions can be used to exponentially stabilize periodic orbits of the hybrid zero dynamics, thereby significantly extending the class of stabilizing controllers. An illustration of this result on a model of a bipedal walking robot is provided.
\end{abstract}

\section{INTRODUCTION AND MOTIVATION}

Hybrid zero dynamics (HZD) was introduced in [24] as a feedback design method that could accommodate underactuation in bipedal robots and thereby move beyond quasi-static, flat-footed walking gaits. At its most basic level, the hybrid zero dynamics is an extension of the Byrnes-Isidori notion of zero dynamics [13], [12] to a hybrid setting, with the additional requirement that the invariant manifolds on which the zero dynamics is defined must also be invariant under the discrete reset maps. Outputs satisfying a vector relative degree [12] condition are designed for each phase of a hybrid model with the objective of creating an exponentially stable periodic orbit in the hybrid zero dynamics [24], [25], [26], [21]. The feedback design is completed by rendering the zero dynamics manifold sufficiently rapidly exponentially attractive [15], [26], [14]. The motivation for assuring a sufficiently fast rate of exponential convergence is that the reset maps can be (and in practice, often are) "expansive", taking a solution that does not lie in the zero dynamics manifold and "pushing" it "further away" from the manifold. Hence, the convergence of the continuous dynamics to the manifold must be sufficiently "attractive" to overcome the "repulsion" of the reset map.

To date, the design of the controller assuring convergence to the zero dynamics manifold has been approached in three

The work of A. D. Ames is supported by NSF grants CNS-0953823 and CNS-1136104, NHARP project 000512-0184-2009 and NASA contract NNX12AB58G, K. Galloway is supported by DARPA Contract W91CRB11-1-0002, and J. Grizzle is supported in part by DARPA and by NSF grant ECS-909300.

A. D. Ames is with the Department of Mechanical Engineering, Texas A\&M University, College Station, TX 77843, email: aamesetamu. edu.

K. Galloway and J. W. Grizzle are with the Control Systems Laboratory, Electrical Engineering and Computer Science Department, University of Michigan, Ann Arbor, MI 48109, email: \{kevinsg,grizzle\}@umich.edu. ways. In [8], Hölder continuous feedbacks based on [5] were used to assure finite-time convergence. In [17], [18], specially selected variables transverse to the zero dynamics manifold are Jacobian linearized and an exponentially stabilizing feedback is computed on the basis of LQR for periodic systems (see also [4] and [19]). In [2], [1], [14], [15], [26] (to only name a few), the transverse dynamics were input-output linearized and a high-gain time-invariant linear feedback was then employed; for example, when the outputs have vector relative degree 2 , after creating a linear relationship between the inputs and outputs, the feedback is chosen to be

$$
u=-\frac{1}{\varepsilon^{2}} K_{P} y-\frac{1}{\varepsilon} K_{D} \dot{y} .
$$

Therefore, the vast body of work that utilizes hybrid zero dynamics for controller design essentially considers PD control on the outputs.

The objective of the present paper is to significantly expand the set of feedback controllers that can be used to render the zero dynamics sufficiently rapidly attractive to exponentially stabilize the periodic orbit in the fulldimensional hybrid model. A control Lyapunov function (CLF) approach is taken, starting in Section II with a review of input-output linearization from a Lyapunov perspective and a subsequent CLF approach to obtaining convergence bounds similar to the linear case. This section culminates with the derivation of the control (28), which is based on Sontag's construction. In Section III we address the more general setting of affine control systems with an invariant zero dynamics surface. The goal is to analyze the full system dynamics in terms of the reduced system dynamics coupled with a CLF which converges sufficiently rapidly in the transverse dynamics. Such a CLF can be used in conjunction with the pointwise min-norm control [6]. The main result of this paper, presented in Section IV, states that for a CLF which is rapidly exponentially stabilizing, the existence of an exponentially stable periodic orbit in the hybrid zero dynamics implies the existence of a feedback rendering the periodic orbit exponentially stable in the full dynamics. Finally, Section V describes simulation results for the CLF-based controllers implemented on a three-link bipedal walker model.

\section{INPUT-OUTPUT LINEARIZATION-A LYAPUNOV PERSPECTIVE}

This section revisits input-output linearization from a Lyapunov perspective in order to motivate the formal constructions of this paper. 


\section{A. Input-Output Linearization Revisited}

To motivate later constructions, we consider an affine control system modeling a mechanical system (with configuration space $\Theta$ and (local) coordinates $\theta \in \Theta$ ) given by

$$
\left[\begin{array}{l}
\dot{\theta} \\
\ddot{\theta}
\end{array}\right]=f_{\theta}(\theta, \dot{\theta})+g_{\theta}(\theta, \dot{\theta}) u
$$

for $(\theta, \dot{\theta}) \in T \Theta$. Suppose that for (1) there is an associated output $y(\theta)$ of vector relative degree 2 on a region of interest (see [16], [11] for a definition and more detailed exposition). This results in

$$
\ddot{y}=L_{f_{\theta}}^{2} y(\theta, \dot{\theta})+L_{g_{\theta}} L_{f_{\theta}} y(\theta, \dot{\theta}) u
$$

where the decoupling matrix, $L_{g_{\theta}} L_{f_{\theta}} y(\theta, \dot{\theta})$, is invertible due to the vector relative degree assumption. Application of an input-output linearizing controller of the form

$$
u(\theta, \dot{\theta})=-\left(L_{g_{\theta}} L_{f_{\theta}} y(\theta, \dot{\theta})\right)^{-1}\left(L_{f_{\theta}}^{2} y(\theta, \dot{\theta})+\frac{1}{\varepsilon^{2}} K_{P} y+\frac{1}{\varepsilon} K_{D} \dot{y}\right),
$$

where $0<\varepsilon<1$ and

$$
A:=\left[\begin{array}{cc}
0 & I \\
-K_{P} & -K_{D}
\end{array}\right]
$$

is Hurwitz, yields the linear system on the outputs

$$
\ddot{y}=-\frac{1}{\varepsilon^{2}} K_{P} y-\frac{1}{\varepsilon} K_{D} \dot{y} .
$$

Choosing the state variables

$$
\eta_{\varepsilon}:=[y / \varepsilon, \dot{y}]^{T}
$$

results in

$$
\varepsilon \frac{d}{d t} \eta_{\varepsilon}(t)=A \eta_{\varepsilon}(t)
$$

and performing a change of time scale $\tau=\frac{t}{\varepsilon}$ yields

$$
\frac{d}{d \tau} \eta_{\varepsilon}(\tau)=A \eta_{\varepsilon}(\tau)
$$

Due to the Hurwitz assumption, for any $Q=Q^{T}>0$ there exists $P=P^{T}>0$ such that the Lyapunov equation is satisfied

$$
A^{T} P+P A=-Q \text {. }
$$

Letting

$$
\gamma:=\frac{\lambda_{\min }(Q)}{\lambda_{\max }(P)}>0,
$$

where $\lambda_{\max }(\cdot)$ and $\lambda_{\min }(\cdot)$ denote (respectively) the maximum and minimum eigenvalues of a given symmetric matrix, we apply the Rayleigh-Ritz inequality to obtain $Q \geq \gamma P$, that is,

$$
A^{T} P+P A+\gamma P \leq 0 .
$$

Then defining the Lyapunov function

$$
V\left(\eta_{\varepsilon}\right)=\eta_{\varepsilon}^{T} P \eta_{\varepsilon}
$$

it follows from (9) that along trajectories of (6) we have

$$
\frac{d V\left(\eta_{\varepsilon}(\tau)\right)}{d \tau} \leq-\gamma V\left(\eta_{\varepsilon}(\tau)\right)
$$

Therefore in the original time-scale (i.e. along trajectories of (5)) we have

$$
\dot{V}\left(\eta_{\varepsilon}(t)\right) \leq-\frac{\gamma}{\varepsilon} V\left(\eta_{\varepsilon}(t)\right),
$$

from which it follows that

$$
V\left(\eta_{\varepsilon}(t)\right) \leq e^{-\gamma t / \varepsilon} V\left(\eta_{\varepsilon}(0)\right) .
$$

Once again employing the Rayleigh-Ritz inequality, from (13) we obtain

$$
\left\|\eta_{\varepsilon}(t)\right\| \leq \sqrt{\frac{\lambda_{\max }(P)}{\lambda_{\min }(P)}} e^{-\frac{\gamma}{2 \varepsilon} t}\left\|\eta_{\varepsilon}(0)\right\|
$$

which leads to

$$
\left\|\begin{array}{l}
y(t) \\
\dot{y}(t)
\end{array}\right\| \leq \frac{1}{\varepsilon} \sqrt{\frac{\lambda_{\max }(P)}{\lambda_{\min }(P)}} e^{-\frac{\gamma}{2 \varepsilon} t}\left\|\begin{array}{c}
y(0) \\
\dot{y}(0)
\end{array}\right\| .
$$

Hence, by choosing $\varepsilon>0$ sufficiently small, the norm of $(y(t), \dot{y}(t))$ converges to zero at a rate inversely proportional to $\varepsilon$.

Remark 1: In the coordinates $\eta_{\varepsilon}=[y / \varepsilon, \dot{y}]^{T}, V$ satisfies

$$
\lambda_{\text {min }}(P)\left\|\eta_{\varepsilon}\right\|_{2}^{2} \leq V\left(\eta_{\varepsilon}\right) \leq \lambda_{\max }(P)\left\|\eta_{\varepsilon}\right\|_{2}^{2},
$$

while in the coordinates $\eta=[y, \dot{y}]^{T}$, we have

$$
\lambda_{\text {min }}(P)\|\eta\|_{2}^{2} \leq V_{\varepsilon}(\eta) \leq \frac{1}{\varepsilon^{2}} \lambda_{\max }(P)\|\eta\|_{2}^{2},
$$

where

$$
V_{\varepsilon}(\eta)=\eta^{T}\left[\begin{array}{cc}
\frac{1}{\varepsilon} I & 0 \\
0 & I
\end{array}\right] P\left[\begin{array}{cc}
\frac{1}{\varepsilon} I & 0 \\
0 & I
\end{array}\right] \eta=: \eta^{T} P_{\varepsilon} \eta .
$$

\section{B. CLF's from Lyapunov Equations}

Our objective is to obtain similar bounds on convergence through control Lyapunov functions, and the goal of this section is to obtain an inequality similar to (12) without a specific choice of controller.

Returning to (2), suppose that a preliminary feedback controller is applied that renders the zero dynamics surface

$$
Z=\left\{(\theta, \dot{\theta}) \in T \Theta \mid y(\theta)=0, L_{f_{\theta}} y(\theta, \dot{\theta})=0\right\}
$$

invariant. An example of such a control law is given by

$$
u(\theta, \dot{\theta})=-\left(L_{g_{\theta}} L_{f_{\theta}} y(\theta, \dot{\theta})\right)^{-1} L_{f_{\theta}}^{2} y(\theta, \dot{\theta})+\mu,
$$

yielding

$$
\ddot{y}=L_{g_{\theta}} L_{f_{\theta}} y(\theta, \dot{\theta}) \mu=: \ell(y, \dot{y}, z) \mu,
$$

where $z \in Z$ represent the uncontrolled states.

Using the coordinates

$$
\eta=\left[\eta_{1}, \eta_{2}\right]^{T}:=[y, \dot{y}]^{T},
$$

the dynamics become

$$
\left[\begin{array}{c}
\dot{\eta}_{1} \\
\dot{\eta}_{2}
\end{array}\right]=\left[\begin{array}{c}
\eta_{2} \\
0
\end{array}\right]+\left[\begin{array}{c}
0 \\
\ell\left(\eta_{1}, \eta_{2}, z\right) \mu
\end{array}\right]
$$

and writing

$$
F=\left[\begin{array}{ll}
0 & I \\
0 & 0
\end{array}\right], \quad G=\left[\begin{array}{l}
0 \\
I
\end{array}\right],
$$


we obtain the "standard" form for a partially linearized system

$$
\dot{\eta}=F \eta+G \ell(\eta, z) \mu:=f(\eta, z)+g(\eta, z) \mu,
$$

where clearly $(F, G)$ is controllable.

We will demonstrate that, subject to the decoupling matrix being invertible, there exists a positive definite differentiable function $V_{\varepsilon}(\eta)$ and a constant $c>0$, such that for all $\varepsilon>0$ and for all $(\eta, z)$, there exists $\mu$ such that

$$
L_{f} V_{\varepsilon}(\eta, z)+L_{g} V_{\varepsilon}(\eta, z) \mu+\frac{c}{\varepsilon} V_{\varepsilon}(\eta) \leq 0 .
$$

In particular, let $\mu$ be given by the input-output linearization control law

$$
\mu(\eta, z)=-\ell(\eta, z)^{-1} K \eta
$$

with $K=\left[\begin{array}{cc}\frac{1}{\varepsilon^{2}} K_{P} & \frac{1}{\varepsilon} K_{D}\end{array}\right]$ so that (20) becomes

$$
\dot{\eta}=f(\eta, z)+g(\eta, z) \mu(\eta, z)=\left[\begin{array}{cc}
0 & I \\
-\frac{1}{\varepsilon^{2}} K_{P} & -\frac{1}{\varepsilon} K_{D}
\end{array}\right] \eta,
$$

which is equivalent to (5). Then defining $V_{\varepsilon}(\eta)$ as in (15) (which is equivalent to (10)), it follows from (12) that

$$
\dot{V}_{\varepsilon}(\eta, z)=L_{f} V_{\varepsilon}(\eta, z)+L_{g} V_{\varepsilon}(\eta, z) \mu(\eta, z) \leq-\frac{\gamma}{\varepsilon} V_{\varepsilon}(\eta),
$$

with

$$
\begin{aligned}
& L_{f} V_{\varepsilon}(\eta, z)=\eta^{T}\left(F^{T} P_{\varepsilon}+P_{\varepsilon} F\right) \eta \\
& L_{g} V_{\varepsilon}(\eta, z)=2 \eta^{T} P_{\varepsilon} G \ell(\eta, z),
\end{aligned}
$$

and therefore (21) is satisfied with $c=\gamma$. More generally, this shows that

$$
\inf _{\mu}\left[L_{f} V_{\varepsilon}(\eta, z)+L_{g} V_{\varepsilon}(\eta, z) \mu+\frac{\gamma}{\varepsilon} V_{\varepsilon}(\eta)\right] \leq 0
$$

since we have produced a specific example of $\mu$ that satisfies this condition. Therefore, $V_{\varepsilon}$ is a control Lyapunov function; moreover, it is a specific example of a stronger form of CLF - a rapidly exponentially stabilizing control Lyapunov function. (A precise definition will be given in Section III.)

While (22) serves as a specific example of a control law which satisfies (21), there exist other control laws which will also satisfy the bound. For example, if we define

$$
\begin{aligned}
& \psi_{0}(\eta, z)=L_{f} V_{\varepsilon}(\eta, z)+\frac{\gamma}{\varepsilon} V_{\varepsilon}(\eta) \\
& \psi_{1}(\eta, z)=L_{g} V_{\varepsilon}(\eta, z)^{T}
\end{aligned}
$$

in terms of (15) and (25), then the "universal" construction of Sontag [20] yields a control law which satisfies (21), given by

$$
\mu(\eta, z)=\left\{\begin{array}{llr}
\Upsilon(\eta, z) & \text { if } & \psi_{1}(\eta, z) \neq 0 \\
0 & \text { if } & \psi_{1}(\eta, z)=0
\end{array}\right.
$$

where

$$
\Upsilon(\eta, z)=-\frac{\psi_{0}(\eta, z)+\sqrt{\left(\psi_{0}(\eta, z)\right)^{2}+\left(\psi_{1}(\eta, z)^{T} \psi_{1}(\eta, z)\right)^{2}}}{\psi_{1}(\eta, z)^{T} \psi_{1}(\eta, z)} \psi_{1}(\eta, z) .
$$

\section{Exponentially StabiLIZING CLF}

In this section we extend our analysis to the broader class of affine control systems of the form

$$
\begin{aligned}
\dot{x} & =f(x, z)+g(x, z) u \\
\dot{z} & =q(x, z),
\end{aligned}
$$

which, for example, models an underactuated mechanical system. Here, $x \in X$ are controlled (or output) states, $z \in Z$ are the uncontrolled states, and $U$ is the set of admissible control values for $u$. In addition, we assume that $f(0, z)=0$, i.e., that the zero dynamics surface $Z$ defined by $x=0$ with dynamics given by $\dot{z}=q(0, z)$ is invariant. (Note that the motivating example in Section II is an example of such a system.) The goal is to understand the dynamics of the full-order system in terms of the dynamics of the reduced-order system coupled with control Lyapunov functions for the $x$-dynamics.

Definition 1: (Based on [28, Def. 3, pp. 4] for discretetime systems.) For the system (29), a continuously differentiable function $V: X \rightarrow \mathbb{R}$ is an exponentially stabilizing control Lyapunov function (ES-CLF) if there exist positive constants $c_{1}, c_{2}, c_{3}>0$ such that

$$
\begin{aligned}
& c_{1}\|x\|^{2} \leq V(x) \leq c_{2}\|x\|^{2} \\
& \inf _{u \in U}\left[L_{f} V(x, z)+L_{g} V(x, z) u+c_{3} V(x)\right] \leq 0
\end{aligned}
$$

for all $(x, z) \in X \times Z$.

In the context of hybrid systems, we will need stronger bounds on convergence than CLF's typically give. This motivates the following definition.

Definition 2: For the system (29), a one-parameter family of continuously differentiable functions $V_{\varepsilon}: X \rightarrow \mathbb{R}$ is said to be a rapidly exponentially stabilizing control Lyapunov function (RES-CLF) if there exist positive constants $c_{1}, c_{2}, c_{3}>0$ such that for all $0<\varepsilon<1$ and for all $(x, z) \in$ $X \times Z$,

$$
\begin{aligned}
& c_{1}\|x\|^{2} \leq V_{\varepsilon}(x) \leq \frac{c_{2}}{\varepsilon^{2}}\|x\|^{2} \\
& \inf _{u \in U}\left[L_{f} V_{\varepsilon}(x, z)+L_{g} V_{\varepsilon}(x, z) u+\frac{c_{3}}{\varepsilon} V_{\varepsilon}(x)\right] \leq 0 .
\end{aligned}
$$

Motivated by [6], in the case of a RES-CLF we can consider the set

$$
K_{\varepsilon}(x, z)=\left\{u \in U: L_{f} V_{\varepsilon}(x, z)+L_{g} V_{\varepsilon}(x, z) u+\frac{c_{3}}{\varepsilon} V_{\varepsilon}(x) \leq 0\right\}
$$

consisting of the control values that result in $\dot{V}_{\varepsilon}(x, z, u) \leq$ $-\frac{c_{3}}{\varepsilon} V_{\varepsilon}(x)$. It is easy to see that for any Lipschitz continuous feedback control law $u_{\varepsilon}(x, z) \in K_{\mathcal{\varepsilon}}(x, z)$, for the resulting closed-loop system

$$
\begin{aligned}
\dot{x} & =f(x, z)+g(x, z) u_{\varepsilon}(x, z) \\
\dot{z} & =q(x, z),
\end{aligned}
$$

it follows (from (32) and (33)) that

$$
V_{\varepsilon}(x(t)) \leq e^{-c_{3} t / \varepsilon} V_{\varepsilon}(x(0))
$$

and

$$
\|x(t)\| \leq \frac{1}{\varepsilon} \sqrt{\frac{c_{2}}{c_{1}}} e^{-\frac{c_{3}}{2 \varepsilon} t}\|x(0)\|
$$


and therefore the exponential convergence can be directly controlled with the constant $\varepsilon$ through $\frac{c_{3}}{\varepsilon}$. Moreover, we can pick the control law of minimum norm in $K_{\varepsilon}(x, z)$

$$
m(x, z)=\operatorname{argmin}\left\{\|u\|: u \in K_{\mathcal{\varepsilon}}(x, z)\right\}
$$

termed the pointwise min-norm control law [6]. For a system of the form (29) with $V_{\varepsilon}$ a RES-CLF, define

$$
\psi_{0}=L_{f} V_{\varepsilon}(x, z)+\frac{c_{3}}{\varepsilon} V_{\varepsilon}(x), \quad \psi_{1}=L_{g} V_{\varepsilon}(x, z)^{T} .
$$

In this case, the pointwise min-norm control law is given by

$$
m(x, z)=\left\{\begin{array}{lll}
-\frac{\psi_{0}(x, z) \psi_{1}(x, z)}{\psi_{1}(x, z)^{T} \psi_{1}(x, z)} & \text { if } \quad \psi_{0}(x, z)>0 \\
0 & \text { if } \quad \psi_{0}(x, z) \leq 0
\end{array}\right.
$$

In the previous section, a specific example of a RES-CLF was given by (15), with $c_{1}=\lambda_{\min }(P), c_{2}=\lambda_{\max }(P)$ and $c_{3}=\gamma$. Picking $\mu(x, z)=m(x, z)$, with $\psi_{0}$ and $\psi_{1}$ defined as above, results in the pointwise min-norm control law such that (21) is satisfied.

\section{CLF'S AND HYBRID SYSTEMS}

This section considers control Lyapunov functions in the context of hybrid systems. Analogous to the case of nonhybrid systems in (29), we will begin with the assumption that the hybrid system already has a hybrid zero dynamics [24], [14], which is a manifold that is invariant under both the continuous and the discrete dynamics of the hybrid system. The goal is to provide conditions for establishing the local exponential stability of a periodic orbit of a hybrid system on the basis of two lower-dimensional problems, namely, establishing the exponential stability of a periodic orbit contained in the hybrid zero dynamics and constructing a control Lyapunov function for the dynamics transverse to the submanifold.

Consider a hybrid control system of the form of a system with impulse effects [3], [27], [10]

$$
\mathscr{H} \mathscr{C}=\left\{\begin{array}{rlrl}
\dot{x} & =f(x, z)+g(x, z) u & \text { if } \quad(x, z) \in D \backslash S \\
\dot{z} & =q(x, z) & & \\
x^{+} & =\Delta_{X}\left(x^{-}, z^{-}\right) \\
z^{+} & =\Delta_{Z}\left(x^{-}, z^{-}\right) & & \text {if } \quad\left(x^{-}, z^{-}\right) \in S
\end{array}\right.
$$

where $x \in X, z \in Z, u \in U$ are defined as in (29), the domain $D$ is an open subset of $X \times Z$, the functions $f, g, q, \Delta_{X}, \Delta_{Z}$ are locally Lipschitz in their arguments, and the guard or switching surface $S \subset D$ is a co-dimension one submanifold of $D$. We assume furthermore that the domain and switching surfaces are given by

$$
\begin{aligned}
D & =\{(x, z) \in X \times Z: h(x, z) \geq 0\} \\
S & =\{(x, z, u) \in X \times Z \times U: h(x, z)=0 \text { and } \dot{h}(x, z)<0\},
\end{aligned}
$$

for some continuously differentiable function $h: X \times Z \rightarrow \mathbb{R}$ for which $L_{g} h=0$. For simplicity of notation, we write $\Delta(x, z)=\left(\Delta_{X}(x, z), \Delta_{Z}(x, z)\right)$ which is the reset map representing the discrete dynamics of the hybrid system.

As previously mentioned, it is assumed that $\mathscr{H} \mathscr{C}$ has a hybrid zero dynamics. More specifically, we assume that $f(0, z)=0$, so that the surface $Z$ is invariant for the continuous dynamics, and that $\Delta_{X}(0, z)=0$, so that the surface $Z$ is invariant for the discrete dynamics. The hybrid zero dynamics is then the hybrid system

$$
\left.\mathscr{H}\right|_{Z}=\left\{\begin{array}{rlrr}
\dot{z} & =q(0, z) & \text { if } & z \in Z \backslash(S \cap Z) \\
z^{+} & =\Delta_{Z}\left(0, z^{-}\right) & \text {if } & z^{-} \in S \cap Z .
\end{array}\right.
$$

For a hybrid system of the form (38) and a RES-CLF $V_{\varepsilon}(x)$, we can again consider locally Lipschitz continuous control laws $u_{\varepsilon}(x, z) \in K_{\varepsilon}(x, z)$. Applying such a control results in a hybrid system

$$
\mathscr{H}_{\varepsilon}=\left\{\begin{array}{rlrl}
\dot{x} & =f(x, z)+g(x, z) u_{\varepsilon}(x, z) & & \text { if } \quad(x, z) \in D \backslash S \\
\dot{z} & =q(x, z) & \\
x^{+} & =\Delta\left(x^{-}, z^{-}\right) & \text {if } \quad\left(x^{-}, z^{-}\right) \in S \\
z^{+} & &
\end{array}\right.
$$

In the context of hybrid systems, one can define solutions in many ways [27], [10], [26], [7]. Because we focus on periodic orbits and solutions that evolve in a neighborhood of such orbits, we are primarily interested in the Poincare map, which can be defined rather easily for the hybrid systems considered here. For the hybrid system $\mathscr{H}_{\varepsilon}$, let $\phi_{t}^{\varepsilon}(x, z)$ be a flow of the continuous dynamics of (41) as given by (34). For $\left(x^{*}, z^{*}\right) \in S$, we say that $\phi_{t}^{\varepsilon}$ is hybrid periodic with period $T>$ 0 if $\phi_{T}^{\varepsilon}\left(\Delta\left(x^{*}, z^{*}\right)\right)=\left(x^{*}, z^{*}\right)$. (Note that here we are assuming that the fixed point is in the switching surface $S$; more general definitions are possible [9], [23], but the one introduced is sufficient for our purposes.) A set $\mathscr{O}$ is a hybrid periodic orbit if $\mathscr{O}=\left\{\phi_{t}^{\varepsilon}\left(\Delta\left(x^{*}, z^{*}\right)\right): 0<t<T\right\}$ for a hybrid periodic flow $\phi_{t}^{\varepsilon}$. (See Fig. 3 for a visual representation.)

Taking $S$ as a Poincaré section, associated with a hybrid periodic orbit is a Poincaré map $P^{\varepsilon}: S \rightarrow S$ which is a partial function

$$
P^{\varepsilon}(x, z)=\phi_{T_{I}^{\varepsilon}(x, z)}^{\varepsilon}(\Delta(x, z)),
$$

where $T_{I}^{\varepsilon}: S \rightarrow D$ is the time-to-impact function defined by

$$
T_{I}^{\varepsilon}(x, z)=\inf \left\{t \geq 0: \phi_{t}^{\varepsilon}(\Delta(x, z)) \in S\right\}
$$

and obtained through the implicit function theorem by considering the function $H(t, x, z)=h\left(\phi_{t}^{\varepsilon}(\Delta(x, z))\right)$ for which $H\left(T, x^{*}, z^{*}\right)=0$. Since, by assumption on $S, \frac{\partial H}{\partial t}\left(T, x^{*}, z^{*}\right)<$ 0 , the implicit function theorem implies that $T_{I}^{\varepsilon}$ is welldefined in a neighborhood of $\left(x^{*}, z^{*}\right)$. Therefore, $T_{I}^{\varepsilon}\left(x^{*}, z^{*}\right)=$ $T$ and so $P^{\varepsilon}\left(x^{*}, z^{*}\right)=\left(x^{*}, z^{*}\right)$. Also, we note that $H(t, x, z)$ is Lipschitz continuous since it is differentiable in $t, h$ is assumed to be continuously differentiable, and $\phi_{t}^{\varepsilon}(\Delta(x, z))$ is Lipschitz continuous, and therefore $T_{I}^{\varepsilon}$ is also Lipschitz [22].

A hybrid periodic orbit, $\mathscr{O}_{Z}$, of $\left.\mathscr{H}\right|_{Z}$ can be similarly defined, in which case the corresponding Poincaré map $\rho$ : $S \cap Z \rightarrow S \cap Z$ (which is again a partial function) is termed the restricted Poincaré map [15]. In this case,

$$
\rho(z)=\phi_{T_{\rho}(z)}^{z}\left(\Delta_{Z}(0, z)\right),
$$

where $\phi^{z}$ is the flow of $\dot{z}=q(0, z)$ and $T_{\rho}(z)$ is the restricted time-to-impact function which is simply given by $T_{\rho}(z):=$ 


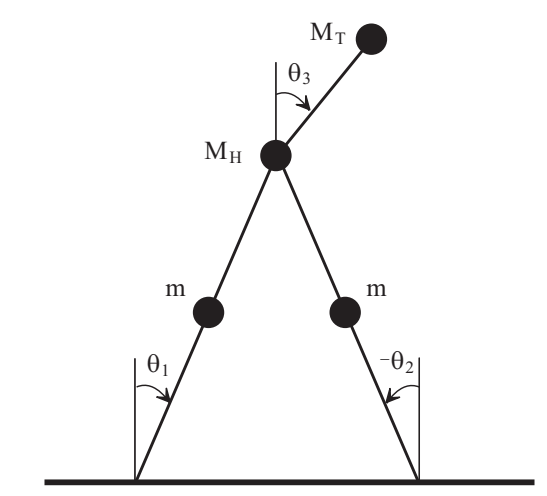

Fig. 1: Model of a three-link bipedal walker.

$T_{I}^{\mathcal{E}}(0, z)$. Due to the assumption that the zero dynamics surface is invariant, a periodic orbit for the zero dynamics, $\mathscr{O}_{Z}$, corresponds to a periodic orbit for the full-order dynamics, $\mathscr{O}=l_{0}\left(\mathscr{O}_{Z}\right)$, through the canonical embedding $\imath_{0}: Z \rightarrow X \times Z$ given by $l_{0}(z)=(0, z)$. With this notation in hand, we present the main result of this paper.

Theorem 1: Let $\mathscr{O}_{Z}$ be an exponentially stable periodic orbit of the hybrid zero dynamics $\left.\mathscr{H}\right|_{Z}$ transverse to $S \cap Z$ and assume there exists a RES-CLF $V_{\varepsilon}$ for the continuous dynamics (29) of $\mathscr{H} \mathscr{C}$. Then there exists an $\bar{\varepsilon}>0$ such that for all $0<\varepsilon<\bar{\varepsilon}$ and for all Lipschitz continuous $u_{\mathcal{E}}(x, z) \in K_{\mathcal{E}}(x, z), \mathscr{O}=\imath_{0}\left(\mathscr{O}_{Z}\right)$ is an exponentially stable hybrid periodic orbit of $\mathscr{H}_{\varepsilon}$.

The proof, which will be published elsewhere, shows that, for the discrete-time dynamical system defined by the Poincare map $P^{\varepsilon}$, the following Lyapunov function

$$
\bar{V}_{\varepsilon}(x, z)=V_{\rho}(z)+\sigma V_{\varepsilon, X}(x)
$$

defined on a neighborhood of $S$ about $(0,0)$ establishes exponential stability, where $V_{\rho}$ is the Lypunov function obtained through the converse-Lyapunov theorem as a result of the exponentially stable discrete-time dynamical system defined by the restricted Poincaré map $\rho, V_{\varepsilon, X}=\left.V_{\varepsilon}\right|_{S}$ for the RES-CLF $V_{\varepsilon}$, and $\sigma>0$ is a positive constant.

\section{Application to Bipedal Walking}

In this section we apply the main result of this paper to the three-link bipedal robot model, as presented in [8]. As described in [8] and displayed in Fig. 1, the model consists of a torso, hip, and two legs (with no feet or knees), with all masses lumped as indicated. Each leg is connected to the hip by a single revolute joint, with torques applied between each leg and the torso, and walking is assumed to take place in the sagittal plane.

We briefly summarize the model construction as follows. Taking the coordinates $\theta=\left(\theta_{1}, \theta_{2}, \theta_{3}\right) \in \Theta$ as illustrated in Fig. 1, the method of Lagrange yields the equations of motion which can be easily rearranged into the form given in (1). (Note that $u$ denotes the two input torques at the hips.) To implement the control designs of Sections II-B and III, we define output functions

$$
y(\theta) \triangleq \theta_{b}-y_{d}\left(\theta_{1}\right)
$$
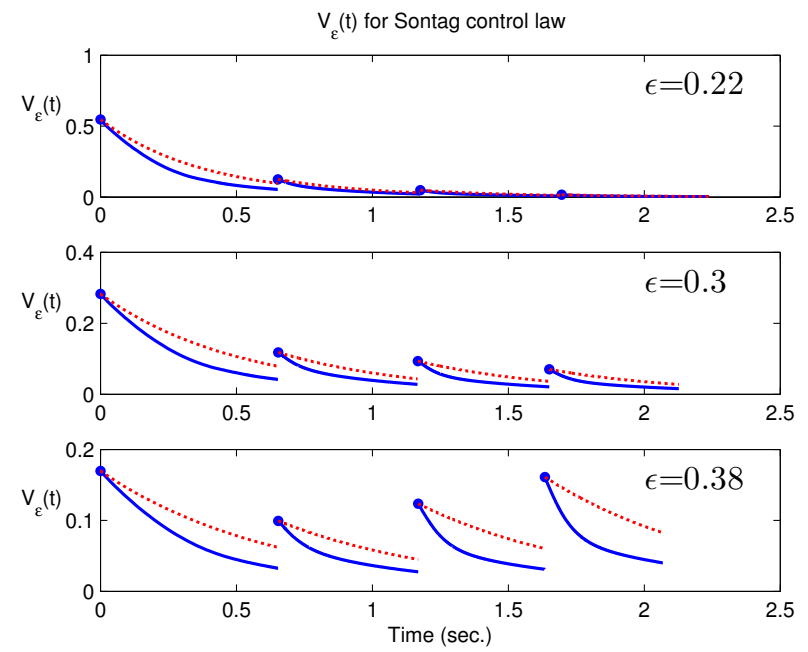

(a) Evolution of the CLF $V_{\varepsilon}(t)$ (solid line) associated with the Sontag controller (28), with the respective nominal bound (35) (dashed line).
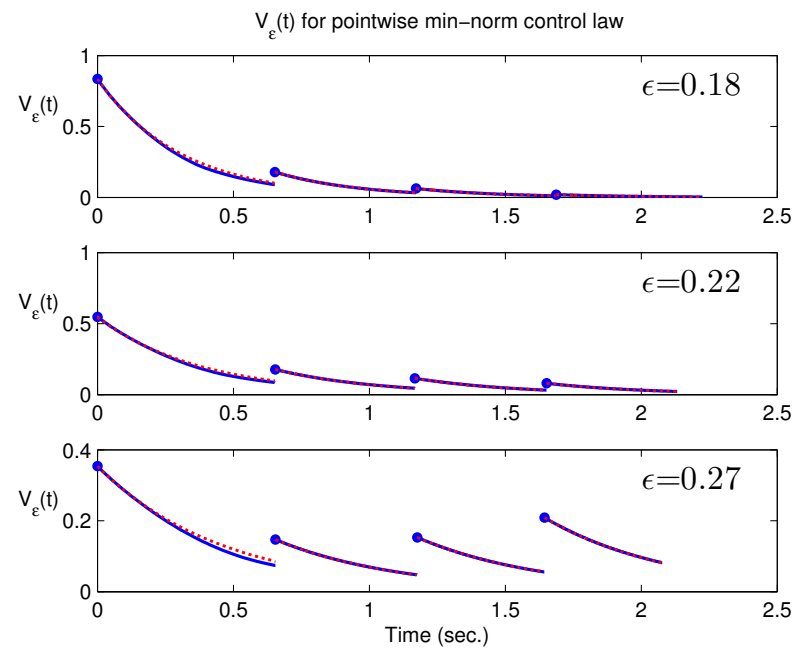

(b) Evolution of the CLF $V_{\varepsilon}(t)$ (solid line) associated with the pointwise min-norm controller (37), with the respective nominal bound (35) (dashed line).

Fig. 2: A four-step simulation started from the same initial condition for the Sontag controller (a), where the top two graphs show $V_{\varepsilon}$ converging to zero (with faster convergence for the smaller $\varepsilon$ value), and the bottom graph shows instability for $\varepsilon$ too large, and for the min-norm controller (b), where $V_{\varepsilon}$ closely follows the nominal bound, only deviating in cases where zero added control (i.e. $\mu(\eta, z)=0$ ) yields convergence which is better than the bound.

where $\theta_{b}=\left(\pi+\theta_{1}-\theta_{3}, \pi+\theta_{2}-\theta_{3}\right)$ are the actuated body coordinates and $y_{d}$ is the desired trajectory of the body coordinates (as a function of $\theta_{1}$ ) over one step. As is described in [24], we parametrize $y_{d}$ as a Bezier polynomial. Also, note that $y(\theta)$ has vector relative degree 2 .

The impulsive nature of walking renders this a hybrid system, with the switching surface $S$ defined by the conditions under which the swing leg impacts the ground in front of the stance leg, i.e., in (39), $h$ corresponds to the height of the 


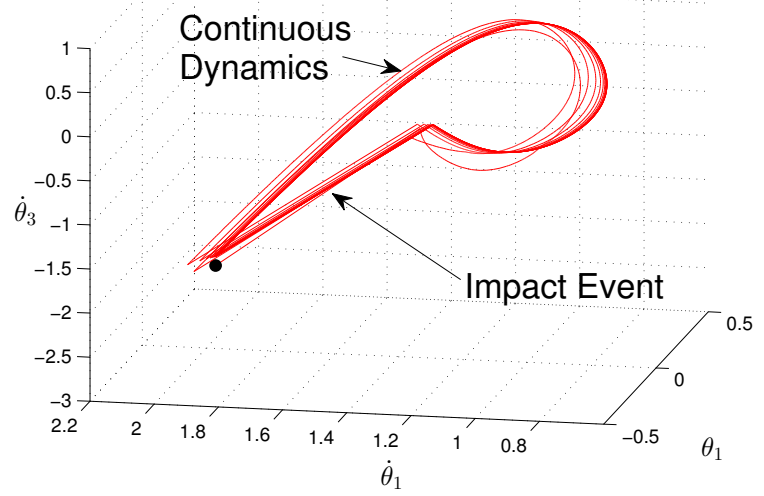

Fig. 3: Convergence to the periodic orbit of $\mathscr{H}_{\varepsilon}$ for the Sontag controller with $\varepsilon=0.1$ over 15 steps for the robot.

foot. In particular, defining $\eta$ as in (17) results in a hybrid control system $\mathscr{H} \mathscr{C}$ that can be expressed in the form of (38) (see [26] for the specific construction). In this case, $\Delta$ describes the change in velocity due to foot strike [8].

The parameters of the Bezier polynomial $y_{d}$ are chosen so that the zero dynamics surface $Z$ (i.e., (16)) is invariant and contains an exponentially stable periodic orbit $\mathscr{O}_{Z}$ transverse to the switching surface. Thus, by design, the assumptions of Theorem 1 are satisfied. In order to render this orbit exponentially stable for the full-order dynamics, we first obtain a RES-CLF $V_{\varepsilon}(\eta)=\eta^{T} P_{\varepsilon} \eta$ as in (15), with $P_{\varepsilon}$ determined by choosing $K_{P}$ and $K_{D}$ and solving the Lyapunov equation (7) for $P$. Then applying either the Sontag control (28) or the pointwise min-norm control (37) results in a hybrid system $\mathscr{H}_{\varepsilon}$ and ensures (by Theorem 1) that the periodic orbit $\mathscr{O}$ is exponentially stable for $\varepsilon$ sufficiently small.

Plots of $V_{\varepsilon}$ and the associated nominal bound (35) for various values of $\varepsilon$ are depicted for the Sontag controller (28) in Fig. 2a and for the pointwise min-norm controller (37) in Fig. 2b. In each case, the middle graph shows slow convergence for one choice of $\varepsilon$; smaller $\varepsilon$ values result in faster convergence (top graphs), and $\varepsilon$ values which are too large result in instability (bottom graphs). Comparing the graphs for $\varepsilon=0.22$ illustrates that the pointwise min-norm controller converges more slowly than the Sontag controller, as is expected. Finally, the convergence to the periodic orbit for the full-order dynamics, as predicted by Theorem 1, can be seen in Fig. 3, where 15 steps of the robot are simulated.

\section{REFERENCES}

[1] A. D. Ames. First steps toward automatically generating bipedal robotic walking from human data. In Robotic Motion and Control, volume 422 of LNICS, pages 89-116. Springer, 2012.

[2] A. D. Ames. First steps toward underactuated human-inspired bipedal robotic walking. In IEEE International Conference on Robotics and Automation, St. Paul, MN, 2012.

[3] D.D. Bainov and P.S. Simeonov. Systems with Impulse Effects : Stability, Theory and Applications. Ellis Horwood Limited, Chichester, 1989.

[4] A. Banaszuk and J. Hauser. Feedback linearization of transverse dynamics for periodic orbits. In Decision and Control, 1994., Proceed- ings of the 33rd IEEE Conference on, volume 2, pages $1639-1644$ vol.2, 1994

[5] S.P. Bhat and D.S. Bernstein. Continuous finite-time stabilization of the translational and rotational double integrators. IEEE Transactions on Automatic Control, 43(5):678-682, 1998.

[6] R. A. Freeman and P. V. Kokotović. Robust Nonlinear Control Design. Birkhäuser, 1996.

[7] R. Goebel, R. Sanfelice, and A. Teel. Hybrid dynamical systems. IEEE Control Systems Magazine, 29(2):28-93, 2009.

[8] J. W. Grizzle, G. Abba, and F. Plestan. Asymptotically stable walking for biped robots: Analysis via systems with impulse effects. IEEE Transactions on Automatic Control, 46(1):51-64, 2001.

[9] J. W. Grizzle, C. Chevallereau, A. D. Ames, and R. W. Sinnet. 3D bipedal robotic walking: models, feedback control, and open problems. In 8th IFAC Symposium on Nonlinear Control Systems, Bologna, Italy, 2010.

[10] W. M. Haddad, V. S. Chellaboina, and S. G. Nersesov. Impulsive and Hybrid Dynamical Systems: Stability, Dissipativity, and Control. Princeton University Press, Princeton, NJ, 2006.

[11] A. Isidori. Nonlinear Control Systems. Springer-Verlag, Berlin, third edition, 1995.

[12] A. Isidori. Nonlinear Control Systems: An Introduction. SpringerVerlag, Berlin, Germany, 3rd edition, 1995.

[13] A. Isidori and C. Moog. On the nonlinear equivalent of the notion of transmission zeros. In Byrnes C. and A. Kurzhanski, editors, Proc. of the IIASA Conference: Modeling and Adaptive Control, pages 146157, Berlin, 1988. Springer-Verlag.

[14] B. Morris and J. W. Grizzle. Hybrid invariant manifolds in systems with impulse effects with application to periodic locomotion in bipedal robots. IEEE Transactions on Automatic Control, 54(8):1751-1764, 2009.

[15] B. Morris and J.W. Grizzle. A restricted Poincaré map for determining exponentially stable periodic orbits in systems with impulse effects: Application to bipedal robots. In IEEE Conf. on Decision and Control, Seville, Spain, 2005.

[16] S. Sastry. Nonlinear Systems: Analysis, Stability and Control. Springer-Verlag, 1999.

[17] A. Shiriaev, J.W. Perram, and C. Canudas-de Wit. Constructive tool for orbital stabilization of underactuated nonlinear systems: Virtual constraints approach. Automatic Control, IEEE Transactions on, 50(8): 1164 - 1176, 2005.

[18] A.S. Shiriaev, L.B. Freidovich, and S.V. Gusev. Transverse linearization for controlled mechanical systems with several passive degrees of freedom. Automatic Control, IEEE Transactions on, 55(4):893 -906, 2010.

[19] G. Song and M. Zefran. Underactuated dynamic three-dimensional bipedal walking. In IEEE International Conference on Robotics and Automation, pages 854-859, Orlando, Florida, 2006.

[20] E. Sontag. A 'universal' contruction of Artstein's theorem on nonlinear stabilization. Systems \& Control Letters, 13:117-123, 1989.

[21] K. Sreenath, H.W. Park, I. Poulakakis, and J. W. Grizzle. A compliant hybrid zero dynamics controller for stable, efficient and fast bipedal walking on MABEL. IJRR, 30(9):1170-1193, 2011.

[22] D. Sun. A further result on an implicit function theorem for locally Lipschitz functions. Operations Research Letters, 28(4):193-198, 2001.

[23] E. Wendel and A. D. Ames. Rank deficiency and superstability of hybrid systems. Nonlinear Analysis: Hybrid Systems, 6:787-805, 2012.

[24] E. Westervelt, J.W. Grizzle, and D.E. Koditschek. Hybrid zero dynamics of planar biped walkers. IEEE Transactions on Automatic Control, 48(1):42-56, 2003.

[25] E. R. Westervelt, G. Buche, and J. W. Grizzle. Experimental validation of a framework for the design of controllers that induce stable walking in planar bipeds. International Journal of Robotics Research, 24(6):559-582, 2004.

[26] E. R. Westervelt, J. W. Grizzle, C. Chevallereau, J. H. Choi, and B. Morris. Feedback Control of Dynamic Bipedal Robot Locomotion. CRC Press, Boca Raton, 2007.

[27] H. Ye, A. N. Michel, and L. Hou. Stability theory for hybrid dynamical systems. IEEE Transactions on Automatic Control, 43(4):461-474, 1998.

[28] W. Zhang, A. Abate, J. Hu, and M. Vitus. Exponential stabilization of dsicrete-time switched linear systems. Automatica, 45(11):2526-2536, 2009. 